\title{
Cancer-Related Fatigue in Adolescents and Young Adults After Cancer Treatment: Persistent and Poorly Managed
}

\author{
Anna Spathis, MSc, ${ }^{1,2}$ Helen Hatcher, PhD, Sara Booth, MD,2 Faith Gibson, PhD, ${ }^{3,4}$ Paddy Stone, MD, \\ Laura Abbas, BSc, Matt Barclay, MSc,2 James Brimicombe, BA, Pia Thiemann, PhD, ${ }^{2}$ Martin G. McCabe, PhD, \\ Rachel Campsey, BSc, Louise Hooker, MSc, Wendy Moss, Jane Robson, BA, and Stephen Barclay, MD²
}

Cancer-related fatigue is the most prevalent and distressing symptom experienced by adolescents and young adults (AYAs). An electronic survey was undertaken to ascertain current fatigue management and perceptions of its effectiveness. Eighty-five percent of responders (68/80) experienced fatigue, and it was worse more than 1 year after cancer treatment ended, compared to $<1$ year $(p=0.007)$. Forty-one percent received no fatigue management. Although advice to exercise was the most frequent intervention, the greatest impact of fatigue was on the ability to exercise and most did not find exercise advice helpful. Early intervention is warranted, supporting AYAs to persevere with increasing activity.

Keywords: fatigue, symptom control, quality of life, late effects

\section{Introduction}

$\mathbf{F}$ ATIGUE IS ONE of the most prevalent, severe, and distressing symptoms experienced by adolescents and young adults (AYAs) with cancer. ${ }^{1,2}$ Healthy adolescents are inherently susceptible to fatigue. The developmental changes of adolescence mean that longer sleep times are needed, while accompanying shifts in circadian rhythm result in later bedtimes. ${ }^{3}$ Sleep is further hindered by use of light-producing electronic devices in the bedroom, social commitments, and alcohol consumption. ${ }^{3,4}$ AYA cancer patients experience even more severe fatigue than their healthy counterparts. ${ }^{5-7}$ Young age and a cancer diagnosis, dual risk factors for fatigue, combine to create a particularly significant problem in this patient group.

Despite this, fatigue is a neglected symptom in AYAs, with no interventional studies focusing on fatigue management at this young age. Research evaluating interventions in older adults has begun to accumulate over the last decade. The most promising approaches are exercise and psychosocial interventions such as education and treatment of concurrent symptoms, with little evidence for pharmacological management. ${ }^{8-11}$ AYAs have unique needs that differ from those of adults and children, and it is well recognized that research outcomes in older adults cannot be extrapolated to the younger population. This has led to an international drive to develop evidence-based age-appropriate care. ${ }^{12,13}$

Preparatory research is needed to support the development of an AYA fatigue intervention that meets their needs. Although qualitative studies have provided insight into the unmet needs of this young group, ${ }^{14-17}$ patients' needs have not been prioritized quantitatively, and there has been no evaluation of the impact on carers. ${ }^{1}$ Furthermore, current practice in fatigue management is unknown. The objectives of this multicenter survey were to quantify the impact of fatigue on young patients and their carers, to find out how fatigue is currently being managed in the United Kingdom, and to ascertain perceptions of the effectiveness of such management.

${ }^{1}$ Cambridge University Hospitals NHS Foundation Trust, Cambridge, United Kingdom.

${ }^{2}$ University of Cambridge, Cambridge, United Kingdom.

${ }^{3}$ Great Ormond Street Hospital for Children NHS Foundation Trust, London, United Kingdom.

${ }^{4}$ University of Surrey, Guildford, United Kingdom.

${ }^{5}$ University College, London, United Kingdom.

${ }^{6}$ University of Manchester, Manchester, United Kingdom.

${ }^{7}$ The Christie NHS Foundation Trust, Manchester, United Kingdom.

${ }^{8}$ University Hospital Southampton NHS Foundation Trust, Southampton, United Kingdom.

(c) Anna Spathis et al. 2017; Published by Mary Ann Liebert, Inc. This is an Open Access article distributed under the terms of the Creative Commons Attribution License, which permits unrestricted use, distribution, and reproduction in any medium, provided the original work is properly cited. 


\section{Methods}

\section{Procedure}

Participants were recruited from three Teenage and Young Adult Principal Treatment Centres (TYA PTCs) in the United Kingdom, and were required to have been diagnosed or treated with cancer at age 13-24 and to be 16-27 at the time of the survey. ${ }^{18}$

Electronic survey methods were used, following advice from TYA PTC service users. After priming by email, poster, and social media, a personalized email of invitation was sent to potential participants, containing a unique token number and links to the Participant Information Sheet and survey. Nonresponders were ascertained by noting unused tokens, and one reminder email was sent to nonresponders after 7 days. Approval was given by the National Research Ethics Service Committee East of England-Cambridgeshire and Hertfordshire (ref. 15/EE/0037).

\section{Outcome measures}

Demographic data were collected from both responders and nonresponders. Participants completed two validated measures. The PedsQL ${ }^{\mathrm{TM}}$ Multidimensional Fatigue Scale (MFS) is a widely used 18-item scale validated in both adolescents and in young adults; scores are linearly transformed to a $0-100$ scale, with lower scores representing worse fatigue. ${ }^{19}$ The Quality of Life Visual Analog Scale (QOL-VAS) is a single-item measure of quality of life that captures a clinically significant change in quality of life more readily than multiple-item measures. ${ }^{20,21}$

The severity of 11 symptoms and the impact of fatigue were quantified using a 5-item verbal rating scale: "not at all," “a little bit," “somewhat," “quite a bit," and "very much." The successfulness of each treatment received for fatigue was rated using a 4-item scale: "entirely successful," "somewhat successful," "helped only a little," and "no effect." A "successfulness score" for fatigue treatment was generated by attributing $0,1,2$, and 3 to the ratings "no effect," "helped only a little," "somewhat successful," and "entirely successful," respectively, for each treatment received and calculating a mean score. The former two ratings defined an unsuccessful treatment and the latter two a successful one. Selection of single or multiple best responses was used for a number of further questions, including defining the two symptoms most affecting daily life, views on the cause of the fatigue, and details of fatigue treatments received.

Participants indicating that fatigue had not affected them over the past month only provided demographic information and recorded the severity of the 11 symptoms, the 2 symptoms most affecting daily life, and the QOL-VAS. SPSS v22 was used for all analyses.

\section{Results}

The survey link was sent to 197 eligible patients and 80 responded, equating to a $41 \%$ response rate. Across all data entry points, $1.8 \%$ were missing.

\section{Characteristics of participants}

There were two main differences between survey responders and nonresponders. First, no patients currently receiving cancer treatment participated. Second, significantly more females than males responded, with $67 \%$ of responders and $47 \%$ of nonresponders being female. However, after excluding patients in the nonresponder group still receiving treatment, to compare responders and nonresponders off

Table 1. Comparison of Responder and Nonresponder Demographic Data

\begin{tabular}{|c|c|c|c|c|c|}
\hline & $\begin{array}{l}\text { Responders } \\
\quad(\mathrm{n}=80)\end{array}$ & $\begin{array}{c}\text { All } \\
\text { nonresponders } \\
(\mathrm{n}=116)\end{array}$ & $\mathrm{p}^{a}$ & $\begin{array}{l}\text { Nonresponders } \\
\text { excluding those on } \\
\text { treatment }(\mathrm{n}=93)\end{array}$ & $\mathrm{p}^{a}$ \\
\hline $\begin{array}{l}\text { Off/on treatment, } \\
n \text { (missing data) }\end{array}$ & $70 / 0(10)$ & $90 / 23(3)$ & $<0.001^{\text {b }}$ & $90 / 0(3)$ & NA \\
\hline $\begin{array}{l}\text { Age at survey in years, } \\
\text { mean (SD, range) }\end{array}$ & $22.1(2.7,17-27)$ & $21.3(2.7,16-27)$ & $0.06^{\mathrm{c}}$ & $21.5(2.6,16-27)$ & $0.21^{\mathrm{c}}$ \\
\hline $\begin{array}{l}\text { Age at diagnosis in years, } \\
\text { mean (SD, range) }\end{array}$ & $18.9(3.1,12-24)$ & $18.2(3.3,9-24)$ & $0.12^{\mathrm{c}}$ & $18.0(3.2,12-24)$ & $0.06^{\mathrm{c}}$ \\
\hline $\begin{array}{l}\text { Months since diagnosis, } \\
\text { median (IQR) }\end{array}$ & $31(18-49)$ & $31(14-58)$ & $0.85^{\mathrm{d}}$ & $37(21-61)$ & $0.19^{\mathrm{d}}$ \\
\hline $\begin{array}{l}\text { Months since last treatment, } \\
\text { median (IQR) }\end{array}$ & $18(10-32)$ & $18(5-46)$ & $0.50^{\mathrm{d}}$ & $27(11-52)$ & $0.05^{\mathrm{d}}$ \\
\hline $\begin{array}{l}\text { Months treatment duration, } \\
\text { median (IQR) }\end{array}$ & $8(4-25)$ & NA & NA & $6(4-9)$ & $0.0066^{\mathrm{d}}$ \\
\hline Gender, $n$, male $(\%)$ & $26(33)$ & $62(53)$ & $0.005^{\mathrm{b}}$ & $44(47)$ & $0.06^{\mathrm{b}}$ \\
\hline \multicolumn{6}{|l|}{ Diagnosis, $n(\%)$} \\
\hline Leukemia & $20(25)$ & $20(17)$ & $0.44^{\mathrm{b}}$ & $12(13)$ & $0.11^{\mathrm{b}}$ \\
\hline Lymphoma & $35(44)$ & $53(46)$ & & $50(54)$ & \\
\hline Osteosarcoma/Ewing's & $6(8)$ & $9(8)$ & & $3(3)$ & \\
\hline Brain neoplasm & $1(1)$ & $8(7)$ & & $6(6)$ & \\
\hline Other & $18(23)$ & $26(22)$ & & $22(24)$ & \\
\hline
\end{tabular}

${ }^{\mathrm{a}} p$-values comparing preceding column of nonresponders with responders and significant $p$-values in bold.

${ }^{\mathrm{b}}$ Fisher's Exact Test.

${ }^{\mathrm{c}}$ Independent samples $t$-test.

${ }^{\mathrm{d}}$ Mann-Whitney U test.

$\mathrm{IQR}$, interquartile range; SD, standard deviation. 


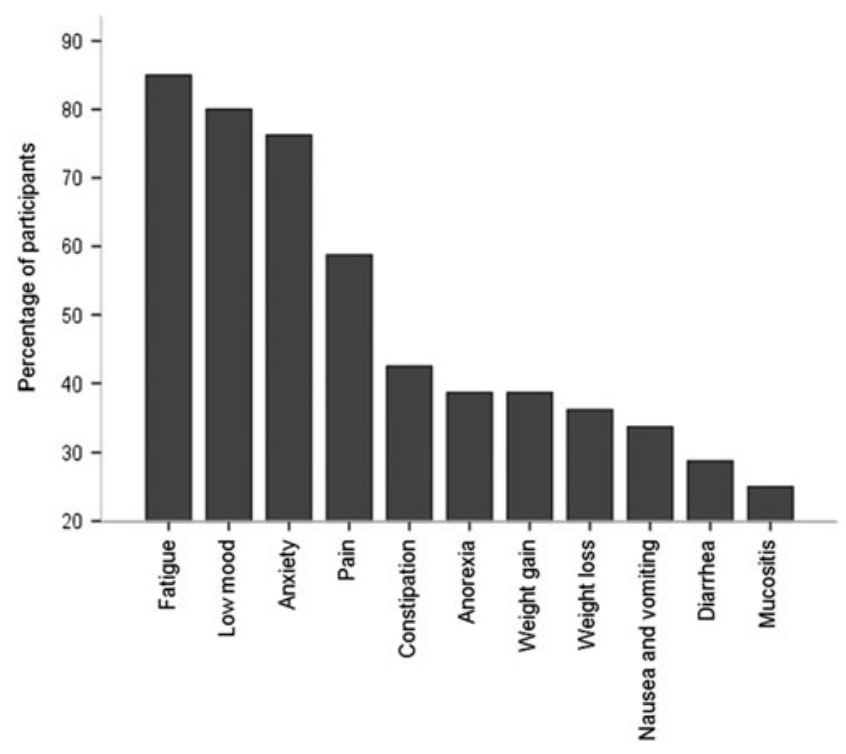

FIG. 1. Symptom prevalence bar chart.

treatment, there was no longer evidence of a gender difference between the two groups (Table 1).

\section{Impact of fatigue}

Fatigue was the most prevalent symptom, experienced by $85 \%$ (68/80) during the preceding month (Fig. 1). Fatigue was ranked as more severe than each of the other symptoms (Wilcoxon signed-rank test; $p<0.001$, except anxiety $p=$ $0.007)$. The mean fatigue severity of the fatigued participants was 44.3 (standard deviation [SD] 20.5). Fatigue severity was worse more than 1 year after the last cancer treatment $(\mathrm{M}=39.0, \mathrm{SD}=19.7)$, compared to $<1$ year $(\mathrm{M}=53.8$, $\mathrm{SD}=19.7$; independent samples $t$-test, $\mathrm{t}(56)=2.8, p=0.007)$. Fatigue was worse in females $(\mathrm{M}=39.6, \mathrm{SD}=19.3)$ than males $(\mathrm{M}=55.6, \mathrm{SD}=19.6 ; \mathrm{t}(66)=3.1, p=0.003)$, but was not associated with other demographic variables, including cancer type or treatment duration. In a linear regression analysis, anorexia and low mood explained $50 \%$ of the variation in fatigue severity (adjusted $\mathrm{R}^{2} 0.50, \mathrm{~F}$ change $=26.60$, $p<0.001)$. Gender no longer had a statistically significant relationship with fatigue after adjusting for anorexia and low mood. Fatigue (MFS) was an independent predictor of quality of life, after adjusting for the potential confounders, low mood, and anorexia (adjusted $\mathrm{R}^{2} 0.42$, F change $=7.13$, $p=0.01)$.

All participants, including those not experiencing fatigue, were asked which 2 out of 11 symptoms most affected daily life. Fatigue was chosen more often than any other symptom (Wilcoxon signed-rank tests, $-7.87<\mathrm{Z}<-4.99$, all $p$-values $<0.001)$. The greatest impact of fatigue on aspects of daily life was on the ability to exercise, with $74 \%$ (50/68) experiencing "somewhat," "quite a bit," or "very much" impact. Attending school or work was hindered for $65 \%$ and socializing for 56\%. Forty-five percent of participants (29/64) felt that their fatigue made the person most involved in their care "somewhat," "quite a bit," or "very much" upset, 42\% (27/ 64) felt it caused frustration, and $41 \%(26 / 64)$ believed it limited rest time. Only $13 \%(8 / 64)$ thought that there was no negative impact.

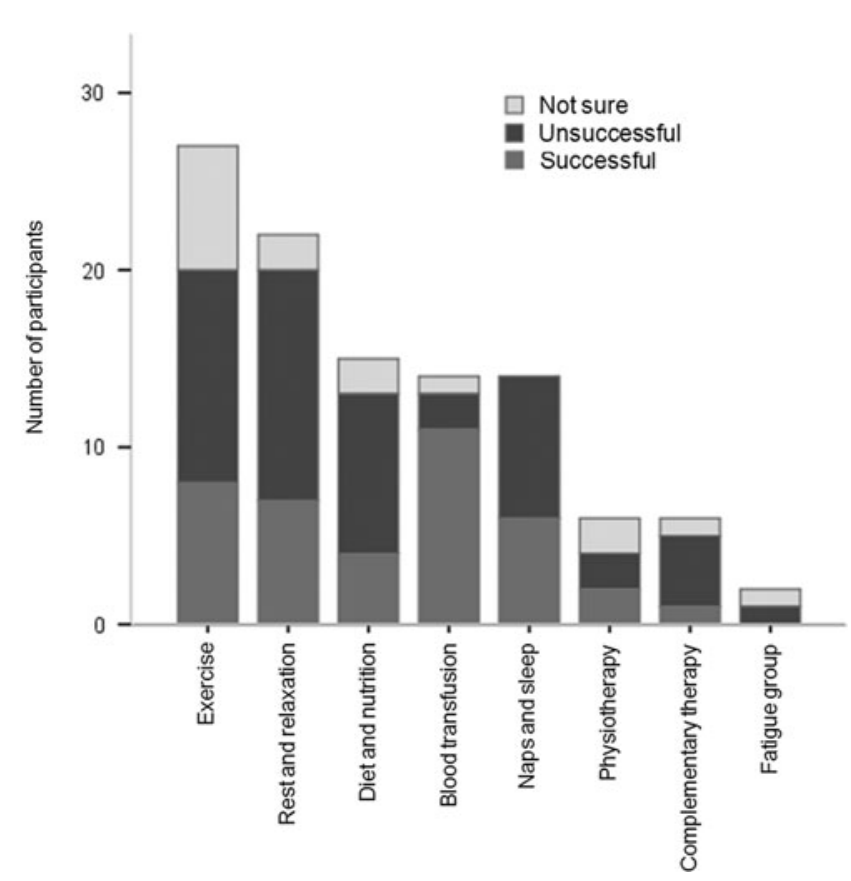

FIG. 2. Stacked bar chart showing successfulness of each fatigue treatment.

\section{Fatigue management}

A minority of fatigued participants $(19 \%, 13 / 68)$ believed that "something could be done" about fatigue, with many (38\%, 26/68) believing it to be a symptom one "has to live with." Although $65 \%$ (44/68) had talked to a health carer about feeling fatigued; the most common reason for not talking was a belief that nothing could be done $(60 \%, 9 / 15)$.

Forty-one percent $(28 / 68)$ of the fatigued participants had not been recommended fatigue treatments. The remaining participants had been recommended a median of 2.5 treatments (range 1-7), most commonly advice to exercise (27/ $40)$ or to rest and relax (22/40). There was no difference in fatigue severity (MFS) between those recommended fatigue treatments and those not. Only blood transfusion was perceived as successful more often than unsuccessful (Fig. 2). Successfulness of fatigue treatment was lower in participants more than 1 year from last cancer treatment compared to those $<1$ year from treatment $[\mathrm{t}(30)=-2.93, p=0.006]$.

All participants, irrespective of whether they had received fatigue treatment, were asked what they personally thought was the single best treatment. Most chose exercise (24\%, 14/ $58)$, followed by diet/nutrition $(12 \%, 7 / 58)$, rest/relaxation $(12 \%, 7 / 58)$, naps/sleeping $(10 \%, 6 / 58)$, blood transfusion $(7 \%, 4 / 58)$, complementary therapies $(5 \%, 3 / 58)$, physiotherapy $(3 \%, 2 / 58)$, and fatigue group attendance $(3 \%, 2 / 58)$. Thirteen of the 16 participants who recommended an active treatment (exercise or physiotherapy) had received an active treatment themselves; those who had received an active treatment were more likely to recommend an active treatment as being better than a rest-focused treatment (Fisher's Exact Test, $p=0.001$ ). However, no evidence was found that those who had received a rest-focused treatment (rest/relaxation, naps/sleeping, or complementary therapy) were more likely to recommend a rest-focused treatment $(p=0.62)$. 


\section{Discussion}

This survey confirms that fatigue is the single most prevalent and severe symptom experienced by AYAs with a cancer diagnosis and has the most negative impact on daily life. Fatigue persisted long after the end of cancer treatment, being perceived as worse more than 1 year after the end of treatment, compared to during the first year following treatment.

Research to date has focused on the burden of fatigue during cancer treatment. Only one study has shown worse fatigue in AYA cancer survivors than controls, ${ }^{7}$ and there have been no longitudinal studies evaluating fatigue changes over time. ${ }^{1}$ One small, cross-sectional observational study has evaluated fatigue according to the time after the end of cancer treatment; there was a suggestion that fatigue in adolescents more than 5 years after treatment $(n=8)$ could be worse than those $1-2$ years after treatment $(n=6) .{ }^{14}$

There may be a number of reasons for the persistence of fatigue well beyond cancer treatment. Resting is counterproductive as the inactivity caused by fatigue leads to a destructive spiral of muscle deconditioning, loss of cardiorespiratory fitness, and worsening fatigue. ${ }^{8,22}$ Furthermore, AYA patients are at a formative age that puts them at particular risk of maladaptive behaviors such as activity avoidance. ${ }^{23}$ Unhelpful health behaviors, such as inactivity, tend to become lifelong habits, increasing the potential for long-term disability. The finding that fatigue severity was worse more than 1 year after the end of treatment may reflect this deconditioning spiral. It could also be that, further from the end of treatment, the expectation that fatigue should be resolving drives a perception of worse fatigue.

These data reveal concerning therapeutic nihilism and under-management of fatigue. Over a decade ago, Stone et al. undertook an influential multicenter survey of older adults with cancer-related fatigue. ${ }^{24}$ While the proportion of people believing that "something could be done" about fatigue remained comparably low (19\% in this survey, $22 \%$ in Stone et al.), there is evidence of progress. Sixty-five percent fatigued participants in this survey had talked to a health carer about their fatigue; $59 \%$ had received some fatigue treatment and, of these, $68 \%$ had received advice to exercise (respective figures in older adults being $48 \%, 14 \%$, and $4 \%) .{ }^{24}$ Exercise was viewed as the best fatigue treatment and was most recommended to others. The fact that most participants found exercise advice unhelpful could be explained by the finding that fatigue has a particularly negative impact on the ability to exercise.

The main limitation of this study is the risk of selection bias. Although a nonresponder analysis was undertaken, fatigue severity was not measured in nonresponders. It is possible that people experiencing fatigue may have been more likely to participate, leading to fatigue overestimation. Greater selection bias further from cancer treatment could have contributed to the findings of worse fatigue and less successful fatigue management in those more than a year after cancer treatment. A longitudinal cohort study, powered to detect changes in fatigue over time, is needed to confirm the study findings.

Nevertheless, this study has a number of implications for clinical practice. The persistence of fatigue, potentially sustained by a spiral of inactivity and deconditioning, suggests that early fatigue intervention is warranted, before maladaptive rest-based behaviors become entrenched at this formative age. Given the impact of fatigue on the ability to exercise, a fatigue intervention must find ways of engaging AYAs in persisting with increasing activity, despite the presence of fatigue. The negative consequences for carers suggest that it would be beneficial to incorporate family support into the intervention.

Considering the high prevalence and detrimental impact of fatigue, it is concerning that there has as yet been no research evaluating age-appropriate interventions. This survey provides evidence for the specific needs of fatigued AYAs and is supporting the design of future research aiming to develop an effective treatment for this important and debilitating symptom.

\section{Acknowledgment}

This research was funded by Macmillan Cancer Support (grant No. 5592228).

\section{Author Disclosure Statement}

No competing financial interests exist.

\section{References}

1. Spathis A, Booth S, Grove S, et al. Teenage and young adult cancer-related fatigue is prevalent, distressing and neglected: it is time to intervene. A systematic literature review and narrative synthesis. J Adolesc Young Adult Oncol. 2015;4(1):3-17.

2. Baggott C, Dodd M, Kennedy C, et al. Changes in children's reports of symptom occurrence and severity during a course of myelosuppressive chemotherapy. J Pediatr Oncol Nurs. 2010;27:307-15.

3. Dahl R, Lewin D. Pathways to adolescent health sleep regulation and behavior. J Adolesc Health. 2002;31(6 Suppl): $175-84$

4. Hysing M, Pallesen S, Morten Stormark K, et al. Sleep and use of electronic devices in adolescence: results from a large population-based study. BMJ Open. 2015;5:e006748.

5. Daniel LC, Brumley LD, Schwartz LA. Fatigue in adolescents with cancer compared to healthy adolescents. Pediatr Blood Cancer. 2013;60:1902-7.

6. Smith AW, Bellizzi KM, Zebrack B, et al. Health-related quality of life of adolescent and young adult patients with cancer in the United States: the adolescent and young adult health outcomes and patient experience study. J Clin Oncol. 2013;31(17):2136-45.

7. Aksnes LH, Hall KS, Jebsen N, et al. Young survivors of malignant bone tumours in the extremities: a comparative study of quality of life, fatigue and mental distress. Support Care Cancer. 2007;15:1087-96.

8. Cramp F, Byron-Daniel J. Exercise for the management of cancer-related fatigue in adults. Cochrane Syst Rev. 2012; (11):Art. No. CD006145.

9. Goedendorp M, Gielissen M, Verhagen C, Bleijenberg G. Psychosocial interventions for reducing fatigue during cancer treatment in adults. Cochrane Syst Rev. 2009;1: CD006953.

10. de Raaf P, de Klerk C, Timman R, et al. Systematic monitoring and treatment in physical symptoms to alleviate fatigue in patients with advanced cancer: a randomised controlled trial. J Clin Oncol. 2013;31(6):716-23.

11. Spathis A, Fife K, Blackhall F, et al. Modafinil for the treatment of fatigue in lung cancer: results of a placebo- 
controlled, double-blind, randomized trial. J Clin Oncol. 2014;32(18):1882-8.

12. National Institute for Health and Clinical Excellence (NICE). Improving outcomes in children and young people with cancer. London: NICE; London: Teenage Cancer Trust, 2005.

13. Teenage Cancer Trust. A blueprint of care for teenagers and young adults with cancer, 2 nd ed. London: Teenage Cancer Trust 2016.

14. Ream E, Gibson F, Edwards J, et al. Experience of fatigue in adolescents living with cancer. Cancer Nurs. 2006;29(4): 317-26.

15. Chiang Y, Yeh C, Wang K, Yang C. The experience of cancer-related fatigue in Taiwanese children. Eur J Cancer Care. 2009;18(1):43-9.

16. McLoone JK, Wakefield CE, Butow P, et al. Returning to school after adolescent cancer: a qualitative examination of Australian survivors' and their families' perspectives. J Adolesc Young Adult Oncol. 2011;1:87-94.

17. Gibson F, Mulhall AB, Richardson A, et al. A phenomenologic study of fatigue in adolescents receiving treatment for cancer. Oncol Nurs Forum. 2005;32(3):651-60.

18. Vindrola-Padros C, Taylor R, Lea S, et al. Mapping adolescent cancer services. Cancer Nurs. 2016;39(5):358-66.

19. Varni JW, Burwinkle TM, Katz ER, et al. The PedsQL in pediatric cancer: reliability and validity of the Pediatric Quality of Life Inventory Generic Core Scales, Multidimensional Fatigue Scale, and Cancer Module. Cancer. 2002;94:2090-2106.
20. de Boer A, van Lanschot J, Stalmeier P, et al. Is a singleitem visual analogue scale as valid, reliable and responsive as multi-item scales in measuring quality of life? Qual Life Res. 2004;13(2):311-20.

21. Huschka M, Mandrekar S, Schaefer P, Jett J. A pooled analysis of quality of life measures and adverse events data in North Central Cancer Treatment Group lung cancer clinical trials. Cancer. 2002;109(4):787-95.

22. Courneya K, Mackey J, Bell G, et al. Randomized controlled trial of exercise training in postmenopausal breast cancer survivors: cardiopulmonary and quality of life measures. J Clin Oncol. 2003;21(9):1660-8.

23. Sawyer S, Afifi R, Bearinger L, et al. Adolescence: a foundation for future health. Lancet. 2012;379(6736): 1630-40.

24. Stone P, Richardson A, Ream E, et al. Cancer-related fatigue: inevitable, unimportant and untreatable? Results of a multi-centre patient survey. Cancer Fatigue Forum. Ann Oncol. 2000;11(8):971-5.

Address correspondence to:

Anna Spathis, MSc

Cambridge University Hospitals NHS Foundation Trust Addenbrookes Hospital Hills Road

Cambridge CB2 8AR United Kingdom

E-mail: aspathis@doctors.org.uk 